\title{
2007/16
}

Games with complementarities

Filippo L. Calciano 


\title{
CORE DISCUSSION PAPER
}

$2007 / 16$

\section{Games with complementarities}

\author{
Filippo L. CALCIANO ${ }^{1}$
}

March 2007

\begin{abstract}
We introduce a class of games with complementarities that has the quasisupermodular games, hence the supermodular games, as a special case. Our games retain the main property of quasisupermodular games: the Nash set is a nonempty complete lattice. We use monotonicity properties on the best reply that are weaker than those in the literature, as well as pretty simple and linked with an intuitive idea of complementarity. The sufficient conditions on the payoffs are weaker than those in quasisupermodular games. We also separate the conditions implying existence of a greatest and a least Nash equilibrium from those, stronger, implying that the Nash set is a complete lattice.
\end{abstract}

Keywords: complementarity, quasisupermodularity, supermodular games, monotone comparative statics, Nash equilibria.

JEL classification: $\mathrm{C} 60, \mathrm{C} 70, \mathrm{C} 72$

${ }^{1}$ CORE, Université catholique de Louvain, Belgium. E-mail: calciano@core.ucl.ac.be

This paper presents research results of the Belgian Program on Interuniversity Poles of Attraction initiated by the Belgian State, Prime Minister's Office, Science Policy Programming. The scientific responsibility is assumed by the author. 


\section{Introduction}

What is the effect on optimal decisions of "complementarities among actions"?

Any attempt to answer this question would rest on what we mean by complementarity. Our practical assesment of two activities being complements, for example tea and lemmon, is that increasing the level of one makes "somehow desirable" to increase the level of the other. Many writers have tried to clarify this loose comparative static idea over the past (Samuelson, 1974, provides for a wide account), and two main groups of definitions of complementariy have arisen: those based on cross-price elesticities of demand, in the Hicks-Allen framework, and those based on mixed second-partials of payoffs, in the Edgeworth-Pareto approach.

In the context of a pure decision problem the first group of definitions would not be appropriate. Markets, hence prices, can not be considered to exist exogenously. They should be obtained as an equilibrium path in a game where society chooses among different allocation mechanisms.

The secong group of definition is more basic, referring only to preferences, and has found a modern equivalent in the notion of supermodularity (Topkis, 1978, but this notion is older) and quasisupermodularity, or ordinal supermodularity (Milgrom and Shannon, 1994).

However, while preferences are certainly a basic datum to study agents, i.e. their attitudes toward risk etc., they are not entirely basic when the object of investigation is directly the solution set of agents' decison problems. Indeed, two games should be considered to be equivalent whenever, up to identifying the strategy spaces in such a way as to take care of duplication of strategies, these games have the same joint best reply.

Hence, to study the effect of complementarities on optimal decisions we should not define complementarities in terms of preferences, but in terms of

This text presents research results of the Belgian Program on Interuniversity Poles of Attraction initiated by the Belgian State, Prime Minister's Office, Science Policy Programming. The scientific responsibility is assumed by the authors. 
the joint best reply correspondence. Then we would need to study the effect of such complementarities on the equilibria.

The intuitive idea of complementarity discussed above may help us to find properties of the joint best reply that could be reasonably assumed as defining complementarities. To introduce these properties, let us restrict the attention for now to games against nature. For example, consider the collection of individual decision problems $\max _{y \in Y} u(y, t)$, where $u: Y \times T \rightarrow \mathbb{R}, Y \subseteq \mathbb{R}^{n}$ and $T \subseteq \mathbb{R}^{m}$. Let $B_{t}:=\operatorname{argmax}_{y \in Y} u(y, t)$.

If, according to our intuitive idea of complementarity, every decision variable $y_{h}$ is a complement with any other decision variable $y_{j}$ and with any parameter $t_{k}$, then we believe it would be reasonable to expect the solution correspondence $B_{t}$ to satisfy at least the following properties for all values $t_{1} \leq t_{2}$ of the parameter:

$$
\begin{aligned}
& \forall s_{1} \in B_{t_{1}}, \exists s_{2} \in B_{t_{2}}: s_{1} \leq s_{2}, \\
& \forall v_{2} \in B_{t_{2}}, \exists v_{1} \in B_{t_{1}}: v_{1} \leq v_{2} .
\end{aligned}
$$

Indeed, consider the first property. If after an increase in the the parameter from $t_{1}$ to $t_{2}$, there is some $s_{1} \in B_{t_{1}}$ such that every $s_{2} \in B_{t_{2}}$ is either strictly less than $s_{1}$ or unordered with $s_{1}$ (the latter meaning that some entries in $s_{2}$ are striclty less than the corresponding entries in $s_{1}$ ) then we should conclude that the increase in the parameter has made somehow desirable to decrease the level of some decision variables (of some entries in $s_{1}$ ), against our intuitive idea of complementarity. The second property is justified analogously.

In this paper we take the properties above, stated on the joint best reply, as the definition of complementarities, and investigate how far we can go with this. A sufficient condition for the joint best reply to satisfy the properties is that every individual best reply satisfy them. We show in Theorem 1 that games whose joint best reply exibiths this kind of monotonicity have, under a weak further condition, a least and a greatest Nash equilibrium. 
Upon strenghtening the monotonicity requirement on the joint best reply, hence with a more stringent complementarity notion, we show in Theorem 2 that the Nash set is a nonempty complete lattice. We then introduce sufficient condition on the payoffs such that the joint best reply is monotonic in the sense required.

Relating to the existing litterature on decisions with complementarities, in this paper we define a class of games that has the quasisupermodular games introduced by Milgrom and Shannon (1994) as a special case. Quasisupermodular games are, in turn, an ordinal extension of supermodular games as introduced in Vives (1990) and Topkis (1979).

Our games retain the main properties of quasisupermodular games: the Nash set is a nonempty complete lattice. Our generalization consists in assuming on the joint best reply correspondence the monotonicity properties introduced above, which are weaker than those required to prove Zhou (1994)'s fixpoint theorem. We also need a weaker structure on the sets of joint best actions. As a result, the sufficient conditions on the payoffs are weaker than those in quasisupermodular games. Our monotonicity properties are pretty intuitive and linked with an intuitive idea of complementarity.

Another difference with the existing literature is that we separate the conditions making the Nash set have a least and a greatest element from those, strongher, making it be a complete lattice.

Our generalization of Zhou's fixpoint theorem, which is in turn an extension to Traski theorem to correspondences, has a very immediate proof. In particular, the lattice subcompleteness of the sets of joint best strategies is completely avoided.

The paper goes as follows. Section 2 introduces the basic terminology of posets and lattices. Section 3 present our generalization of Zhou's fixpoint theorem and presents fixpoint theorems for corresponences on posets. Section 4 introduces and studies our class of games with complementarities. 


\section{Background}

We define here the basic objects that we need in the paper. The most important notions are those of maximal element and complete lattice.

Let $X$ be a nonempty set. A partial order on $X$ is a reflexive, antisymmetric and transitive binary relation $\leq$ on $X$. The set $X$ togheter with $\leq$ is called a partially ordered set, or a poset. We define on $X$ the binary relation $<$ by means of $x<y$ iff $x \leq y$ and not $y \leq x$, for every $x, y \in X$. We define on $X$ the relation "is unordered with" by means of $x$ "is unordered with" $y$ iff neither $x \leq y$ nor $y \leq x$, for every $x, y \in X$.

Take any nonempty subset $S \subseteq X$. An element $x \in X$ is an upper bound of $S$ if every $s \in S$ is a predecessor of $x$ with respect to $\leq$; that is, if $s \leq x$ for every for every $s \in S$. An element $x \in X$ is a supremum of $S$ if it is an upper bound of $S$ and, for any element $y \in X$ which is an upper bound of $S, x \leq y$. An element $x \in X$ is a greatest element of $S$ if it is an upper bound of $S$ and $x \in S$. Suprema and greatest elements are unique $(\bmod =)$ by antisymmetry of $\leq$, and are denoted by $\sup S$ and $1_{S}$ respectively.

Note that $\sup S \leq 1_{s}$, with equality holding iff $\sup _{S} \in S$.

An element $x \in X$ is a maximal element of $S$ if $x \in S$ and, for every $s \in S$, either $s \leq x$ or $s$ is unordered with $x$. Clearly $x \in S$ is a maximal element of $S$ if and only if for every $s \in S, x \leq s$ implies that $x=s$. Maximal elements are not necessarily unique. If $S$ has a greatest element $1_{S}$, it is the unique maximal element of $S$.

The dual poset of $X$ is the set $X$ endowed with the partial order $\geq$ defined by $x \geq y$ iff $y \leq x$, for every $x, y \in X$. Lower bounds of $S$, the infimum inf $S$, the least element $0_{S}$ and the minimal elements of $S$ are defined dually; that is, they are respectively the upper bounds, the supremum, the greatest element and the maximal elements of $S$ considered as a subset of the dual of $X$.

If supS exists, it is the least element of the set of upper bounds of $S$ or, equivalently, the infimum of the set of upper bounds of $S$. The supremum and the infimum of the empty set of $X$, if they exist, are respectively the 
least and the greatest element of $X$. They are denoted by 0 and 1 .

For any subset $T \subseteq S$, the upper bounds of $T$ in $S$ are the upper bounds of $T$ (in $X$ ) which belong to $S$. The restriction of $\leq$ to $S$ is still a partial order, called the induced order on $S$. Thus we can define the supremum of $T$ in $S$, denoted by $\sup _{S} T$, as the least element of the set of upper bounds of $T$ in $S$. Since the set of upper bound of $T$ is $S$ is a subset of that of upper bounbs of $T$, one has $\sup T \leq \sup _{S} T$ whenever both of these exist.

The closed intervals of a poset $X$ are the sets $[x,+\infty):=\{z \in X: x \leq z\}$, $(-\infty, x]:=\{z \in X: z \leq x\}$, and $[x, y]:=\{z \in X: x \leq z \leq y\} ;$ with $x, y \in$ $X$. If $T \subseteq S \subseteq X$ and $\sup T$ exists, then $\sup _{S} T$ whenever it exists is the least elements of $S \cap[\sup T,+\infty)$.

Note that if a poset $X$ has a 1 , then $[x,+\infty)=[x, 1]$ for every $x$ in $X$. If $X$ has a 0 , then $(-\infty, x]=[0, x]$ for every $x \in X$.

If $Y$ is a poset and for every $x, y \in Y$ either $x \leq y$ or $y \leq x$, then $Y$ is called a chain. A chain of a poset $X$ is a subset $S \subseteq X$ which is a chain in the induced order on $S$. An ascending chain of $X$ is a chain of $X$ having a least element. A descending chain of $X$ is an ascending chain of the dual of $X$. If $x \in X$ is the least (greatest) element of an ascending (descending) chain of $X$, we say that this ascending (descending) chain "starts" at $x$.

A poset $X$ is a join lattice if every nonempty and finite subset of $X$ has a supremun. In a join lattice every subset (not necessarily finite) whose set of lower bounds is nonempty and finite has an infimun, the latter being the supremun of such set of lower bounds. $X$ is a meet lattice if its dual is a join lattice; that is, if every finite nonempty subset of $X$ has an infimum. $X$ is a lattice if both $X$ and its dual are join lattices; that is, if $X$ has all nonempty finite suprema and infima.

$X$ is a join-complete lattice if every nonempty subset of $X$ (not necessarily finite) has a supremum. A join-complete lattice has a 1, the supremum of $X$ itself, and it has the infimum of all subsets of $X$ whose set of lower bounds is nonempty. $X$ is a meet-complete lattice if its dual is a joincomplete lattice, or equivalently if every nonempty set of $X$ has an infimum. 
A meet-complete lattice has a 0 , the infimum of $X$ itself.

$X$ is a complete lattice if every subset of $X$, including the empty set, has a supremum. A complete lattice is a join-complete lattice with a 0 , which is by definition the supremum of the empty set of $X$. Clearly, $X$ is a complete lattice iff it is both join-complete and meet-complete, or equivalenty iff every nonempty subset of $X$ has both a supremum and an infimum.

A subset $S$ of a poset $X$ is a complete lattice whenever it is so in the induced order; that is, whenever for every nonempty subset $T \subseteq S, \sup _{S} T \in$ $S$ ( $S$ is join-complete) and $S$ has a least element $0_{S}$. A subset $S$ of a lattice $X$ is a sublattice of $X$ whenever for every nonempty and finite subset $T \subseteq S$, supT $\in S$. A subset $S$ of a complete lattice $X$ is a subcomplete sublattice of $X$ if for every nonempty subset $T \subseteq S$, not necessarily finite, supT $\in S$ and $S$ has a least element $0_{S}$. A subcomplete sublattice of $X$ is also called a closed sublattice of $X$. If $S$ is a subcomplete sublattice of $X$ then it is a complete lattice. The converse is false.

If $X$ is a complete lattice, all of its closed intervals $[x, 1],[0, x],[x, y]$, with $x, y \in X$, are subcomplete sublattices of $X$.

Clearly the intersection of any family of subcomplete sublattices of a complete lattice $X$ is a subcomplete sublattice of $X$. However, the intersection of a subcomplete sublattice of $X$ with a subset of $X$ which is a complete lattice, needs not to be even a complete lattice. Take for example the subcomplete sublattice $[2,3] \subset \overline{\mathbb{R}}$ and the complete lattice $[2,3) \cup\{4\} \subset \overline{\mathbb{R}}$. The intersection is $[2,3)$, which lacks a greatest element.

\section{Fixpoint theorems for increasing correspon- dences.}

Let $X$ be a poset and $F: x \in X \mapsto F_{x} \subseteq X$ be a nonempty correspondence. We associate to $F$ the sets

$$
A_{F}:=\left\{x \in X: F_{x} \cap[x,+\infty) \neq \emptyset\right\},
$$




$$
\begin{gathered}
B_{F}:=\left\{x \in X: F_{x} \cap(-\infty, x] \neq \emptyset\right\}, \\
E_{F}:=\left\{x \in X: x \in F_{x}\right\} .
\end{gathered}
$$

$E_{F}$ is the fixpoint set of $F$. Our concern in this paper is to study the nonemptiness and order structure of $E_{F}$ for a given, nonempty $F$.

$A_{F}$ is the set of points $x \in X$ such that some $y \in F_{x}$ is "above the diagonal". $B_{F}$ is the set of points $x \in X$ such that some $y \in F_{x}$ is "below the diagonal". Of course $E_{F}=A_{F} \cap B_{F}$. Hence, if any among $A_{F}$ and $B_{F}$ is empty $F$ cannot have fixpoints. We thus consider, without loosing generality, only correspondences $F$ with nonempty $A_{F}$ and $B_{F}$. A sufficient condition for all nonempty $F$ to satisfy this would be to assume that $X$ has a 0 and a 1 , respectively.

Let $X$ be a lattice. We say that a correspondence $F: X \rightarrow X$ is Veinottincreasing $^{1}$ if for every $x, y \in X, x \leq y$ implies that for any $u \in F_{x}$ and any $v \in F_{y}$, (a) inf $\{u, v\} \in F_{x}$, called meet increasingness, and (b) $\sup \{u, v\} \in F_{y}$, called join increasingness.

We report, for the sake of completeness, Zohu (1994, Theorem 1) extension of Tarski fixpoint theorem to Veinott-increasing correspondences. Zhou's theorem is a crucial ingredient in the theory of supermodular games.

Theorem 0 (Zohu): Let $X$ be a nonempty complete lattice and $F$ : $X \rightarrow X$ be a nonempty correspondence. If $F$ is Veinott-increasing and $F_{x}$ is a subcomplete sublattice of $X$ for every $x \in X$, then the fixpoint set of $F$ is a nonempty complete lattice.

\subsection{Increasingness notions}

We propose the following notion of increasingness, by which we will generalize the existence part of Zhou's fixpoint theorem (1994, Theorem 1, (i) and (ii))².

Definition 1 (increasing correspondence): Let $X$ be a poset. A

\footnotetext{
${ }^{1}$ See for example Topkis (1978) for the attribution of this notion of increasingness to Veinott

${ }^{2}$ Suggestions for better names than those in Definitions 1 and 2 are welcomed.
} 
nonempty correspondence $F: X \rightarrow X$ is upper increasing if for every $x, y \in$ $X, x \leq y$ implies that

$$
\forall s \in F_{x}, \exists t \in F_{y}: s \leq t
$$

It is lower increasing if $\forall x, y \in X, x \leq y$ implies that

$$
\forall v \in F_{y}, \exists u \in F_{x}: u \leq v
$$

It is increasing if it is both upper and lower increasing.

Intuitively, upper increasingness amounts to say that if $x \leq y$, then for any $s \in F_{x}$ there is in $X$ some ascending chain starting at $s$ which crosses $F_{y}$ at least finitely many times; in other words we can extend any point in $F_{x}$ to at least one point of $F_{y}$ throught an ascending chain. Symmetrically, lower increasingness amounts to say that whenever $x \leq y$, for any $v \in F_{y}$ there is some descending chain in $X$ starting at $v$ which crosses $F_{x}$. If $F$ is a function, upper increasingness and lower increasingness are equivalent and each one of them coincides with $F$ being increasing in the usual sense; namely, $x \leq y$ implies $F(x) \leq F(y)$ for every $x, y \in X$.

On lattices, this notion of increasingness is clearly weaker than Veinott's increasingness. Indeed, if $F$ is Veinott increasing and $x \leq y$, then for any $s \in F_{x}$ and and $v \in F_{y}, \sup \{s, v\}$ is the required $t$ in (1), hence $F$ is upper increasing, and $\inf \{s, v\}$ is the required $u$ in (2), hence $F$ is lower increasing.

On the other hand, a correspondence may well be increasing without being Veinott-increasing. We give the easiest example.

Example 1: A correspondence which is increasing but not Veinottincreasing. Take the six-point (complete) lattice

$$
X:=\{0,1, b, c, \inf \{b, c\}, \sup \{b, c\}\}
$$

with 0 and 1 being respectively the least and greatest element. The only 
unordered elements are $b, c$. Take the correspondence $F: X \rightarrow X$ defined by the identity at the points $0, c, 1$; and by $F_{\text {inf }\{b, c\}}=\{0\}, F_{b}=\{0, b\}$, and $F_{\text {sup }\{b, c\}}=\{c, 1\}$. Such an $F$ is both upper and lower increasing, but it is neither join increasing nor meet increasing, since for $b \leq \sup \{b, c\}$, taking $b \in F_{b}$ and $c \in F_{\text {sup }\{b, c\}}, \sup \{b, c\} \notin F_{\text {sup }\{b, c\}}$ and inf $\{b, c\} \notin F_{b}$.

Note also that in this example any $F_{x}$ is a subcomplete sublattice and that the fixpoint set of $F$ is $\{0,1, b, c\}$, which is a complete lattice (albeit not a sublattice of $X)$. This proves that Veinott increasingness is not necessary in Zhou's theorem.

To generalize the "structure" part of Zhou fixpoint theorem (1994, Theorem 1, (iii)), we will need to single out, for correspondences, another property which is always satisfied by increasing functions. Definition 2 below provides for this. We need some preliminary notation.

For a poset $X$ and a nonempty correspondence $F: X \rightarrow X$, for any $a \in X$, let $U^{a}:=\left\{x \in X: F_{x} \cap[a,+\infty) \neq \emptyset\right\}$. If $F$ is a function, $U^{a}$ is just the upper contour set of $F$ at $a \in X$.

For every $a \in X$ such that $U^{a}$ is nonempty, define the nonempty correspondence $G^{a}: U^{a} \rightarrow[a,+\infty)$ by $G_{x}^{a}=F_{x} \cap[a,+\infty)$. If $F$ is a function, $G^{a}$ is jut the restriction of $F$ to $U^{a}$. The role of $G^{a}$ is to retain, from every $F_{x}$, just and only the elements which are greater than or equal to $a$, whenever any does exist, i.e. whenever $x$ is in $U^{a}$.

Definition 2 (strongly lower increasing correspondence): Let $X$ be a poset. A nonempty correspondence $F: X \rightarrow X$ is strongly lower increasing if, for every $a \in X$ such that $U^{a} \neq \emptyset$, the correspondence $G^{a}$ is lower increasing.

Intuitively, strong lower increasingness says that $x, y \in X$ are such that both $F_{x}$ and $F_{y}$ have some elements which "jumps above" some common $a \in X$, and if $x \leq y$, then for any $v$ in $F_{y}$ which is above $a$, we can find in $X$ a descending chain starting at $v$ and crossing $F_{x}$ still above $a$.

On setting $a=-\infty$, one has that $U^{a}$ is $X$ and that $G^{a}$ is $F$. Hence 
strong lower increasingness implies lower increasingness. If $F$ is a function, the opposite is also true, and so for functions upper increasingness, lower increasingness and strong lower increasingness all boil down to increasingness in the usual sense.

Example 2: A correspondence which is increasing but not strongly lower increasing. In the previous example $F$ is not strongly lower increasing. Indeed, for any $a$ in $\{0,1, b, c$, inf $\{b, c\}, \sup \{b, c\}\}, U^{a}$ is nonempty. $G^{0}$ is $F$, and so it is lower increasing. $G^{1}, G^{b}, G^{\text {sup }}$ are all increasing functions, and so they are lower increasing. $G^{c}$ coincides with the restriction of $F$ to $U^{c}$, and so it is lower increasing. But on $U^{\text {inf }}=\{b, c, \sup \{b, c\}, 1\}$, we have that $G_{b}^{\text {inf }}=\{b\}$ and $G_{\text {sup }}^{\text {inf }}=\{c, 1\}$. Thus for $c \in G_{\text {sup }}^{\text {inf }}$, there is no $u \in G_{b}^{\text {inf }}$ such that $u \leq c$.

Over lattices, strong lower increasingness is obviously implied by meet increasingness, hence by Veinott increasingness. Indeed, take any $a \in X$ such that $U^{a}$ is nonempty and take any $x, y \in U^{a}$ such that $x \leq y$. Pick any $z \in G_{x}^{a}$ and any $v \in G_{y}^{a}$. By meet-increasingness, inf $\{z, v\} \in F_{x}$, and since $a$ minorizes $\{z, v\}$ in $X, a \leq \inf \{z, v\}$. Thus inf $\{z, v\}$ is in $G_{x}^{a}$, and it is the required $u$ in (2). The converse is false. A corresppondence can be strongly lower increasing without being meet increasing, as the following examples show.

Example 3.1: A correspondence which is strongly lower increasing but not Veinott-increasing. Take the five-point (complete) lattice

$$
X:=\{0,1, b, c, d\}
$$

where $d<c, b, c$ are unordered, $b, d$ are unordered, 0 is the least element and 1 is the greatest element. Note that $\inf \{b, c\}=\inf \{b, d\}=0$, and $\sup \{b, c\}=\sup \{b, d\}=1$. Consider the correspondence $F: X \rightarrow X$ definded by the identity at $0, c, d$, and by $F_{b}=\{b, d\}$, and $F_{1}=\{c, 1\}$. This correspondence is increasing but fails to be Veinott-increasing. Indeed, 
for $b \leq 1, b \in F_{b}$ and $c \in F_{1}$, but inf $\{b, c\}=0 \notin F_{b}$. Hence $F$ fails to be meet increasing. On the other hand, $F$ is strongly lower increasing. In fact, for any $a$ in $\{0,1, b, c, d\}, U^{a}$ is nonempty. $G^{0}$ is $F$, and so it is lower increasing. $G^{1}$ and $G^{b}$ are increasing functions, and so they are lower increasing. $G^{c}$ is the restriction of $F$ to $U^{c}$, and so it is lower increasing. Finally, on $U^{d}=\{b, c, d, 1\}$, the correspondence $G^{d}$ coincides with $F$ except at $b$, where $G_{b}^{d}=\{d\}$. Examining the behavior of $G^{d}$ on the two chains of $U^{d}$, namely $b<1$ and $d<c<1$, one sees that $G^{d}$ is in fact lower increasing. The role of the correspondences $G^{a}$ has been to remove from $F_{b}$ the point $b$, whose presence caused $F$ not to be meet increasing.

Example 3.2: A correspondence which is strongly lower increasing but not Veinott-increasing. Let $B$ be the unit square in $\mathbb{R}^{2}$ and, for any $a=\left(a_{1}, a_{2}\right) \in B$, let $F_{a}$ be the intersection of $B$ with the closed triangular region with vertices at $\left(a_{1}, a_{2}\right),\left(a_{1}-\epsilon, a_{2}+\epsilon\right),\left(a_{1}+\epsilon, a_{2}+\epsilon\right)$, for a fixed $\epsilon>0$. The correspondence $F$ so defined maps a complete lattice into convex compact subsets of itself (drawing some pics may help the reader).

Pick $x, y$ in $B$ such that the following holds: $x<y, F_{x}$ and $F_{y}$ are disjoint, and $y_{2}<x_{2}+\epsilon$ (adjust $\epsilon$ if necessary and work with the corresponding $F)$. The vertex of $F_{x}$ with coordinates $\left(x_{1}+\epsilon, x_{2}+\epsilon\right)$ is unordered with the vertex of $F_{y}$ with coordinates $\left(y_{1}, y_{2}\right)$, because $x_{1}+\epsilon<y_{1}$ since $F_{x}$ and $F_{y}$ are disjoint and $y_{2}<x_{2}+\epsilon$. The infimum of these two vertices is the point with coordinates $\left(x_{1}+\epsilon, y_{2}\right)$, which does not belong to $F_{x}$. Thus $F$ fails to be meet increasing, hence to be Veinott-increasing.

On the other hand, first note that for every $a \in B, a \in U^{a}$. Furthermore, for every $z \in U^{a}, z \in G_{z}^{a}=F_{z} \cap[a, 1]$. Fix then any $a$ and pick some $x, y \in U^{a}$ such that $x<y$. If $F_{x}$ and $F_{y}$ are disjoint, then by construction for every $v \in G_{y}^{a}, x \leq v$ and we are done since $x \in G_{x}^{a}$. If $F_{x}$ and $F_{y}$ are not disjoint, for every $v \in G_{y}^{a}$, by construction at least one among the two straight lines throught $v$ and parallel respectively to the orizontal and the vertical axis intersects $G_{x}^{a}$. Take any $u$ in one of these intersections. We have $u \leq v$, and we are done. Hence $F$ is strongly lower increasing. 


\subsection{Fixpoint theorems}

Theorem 1 below generalizes the existence part of Zhou's fixpoint theorem (1994, Theorem 1, points (i) and (ii)) in two repects. First, it requires the correspondence $F$ to be increasing, instead of Veinott increasing. Second, it requires for any $x \in X$ that $F_{x}$ has a least and a greatest element, instead of requiring it to be a subcomplete sublattice of $X$.

Observe also that in Theorem 1, similarly to Tarski theorem, the completeness of the lattice $X$ would play only the role of making $\sup A_{F}$ and inf $B_{F}$ exist, while in Zhou's theorem it is a crucial ingredient in the proof. To underscore this difference, we state Theorem 1 in the context of posets.

Similarly to Zhou's theorem, also Theorem 1 follows from Tarski theorem by an immediate increasing selection argument. The role of Theorem 1 is then to extend Tarski theorem to correspondence in a similar vein as Kakutani's fixpoint theorem extends Brower's.

Theorem 1: Let $X$ be a poset and $F: X \rightarrow X$ be a nonempty correspondence. (i) If $F$ is upper increasing and $F_{x}$ has a greatest element for every $x \in X$, then sup $A_{F}$, whenever it exists, is the greatest fixpoint of $F$. (ii) If $F$ is lower increasing and $F_{x}$ has a least element for every $x \in X$, then inf $B_{F}$, whenever it exists, is the least fixpoint of $F$.

Proof: (i) Let $x^{*}:=\sup A_{F}$. It exists by assumption. Pick any $x \in A_{F}$. By the definition of $A_{F}$, there exists $y_{x} \in F_{x}$ such that $x \leq y_{x}$. Since $x \leq x^{*}$ and $y_{x} \in F_{x}$, by upper increasingness of $F$ there exists $z_{x} \in F_{x^{*}}$ such that $y_{x} \leq z_{x}$. Hence, for the greatest element $1^{*}$ of $F_{x^{*}}, x \leq y_{x} \leq z_{x} \leq 1^{*}$. Since $1^{*}$ does not depend on the chosen $x$, then for every $x \in A_{F}, x \leq 1^{*}$. Thus $1^{*}$ majorizes $A_{F}$ and so $x^{*} \leq 1^{*}$, because $x^{*}$ is the sup of $A_{F}$. Hence, by the definition of $A_{F}$ and because $1^{*} \in F_{x^{*}}, x^{*} \in A_{F}$. So $x^{*}$ is the greatest element of $A_{F}$.

Since $x^{*} \leq 1^{*}$ and $1^{*} \in F_{x^{*}}$, then by upper increasingness of $F$ there exists some $y \in F_{1^{*}}$ such that $1^{*} \leq y$. Hence, by the definition of $A_{F}, 1^{*} \in A_{F}$, and because $x^{*}$ is the greatest element of $A_{F}, 1^{*} \leq x^{*}$. Hence $x^{*}=1^{*} \in F_{x^{*}}$, and 
$x^{*}$ is a fixpoint of $F$. Since $E_{F} \subseteq A_{F}$, where $E_{F}$ is the fixpoint set of $F$, and since $x^{*}$ is the greatest element of $A_{F}$, then $x^{*}$ is indeed the greatest fixpoint of $F$.

(ii) Redo the proof of (i) taking the dual of $X$, and dualizing the statements by substituting $B_{F}$ for $A_{F}$, lower increasingness for upper increasingness, and by using the assumption that $F_{x}$ has a least element for every $x \in X$. Q.E.D

One might be interested in the existence of fixpoints for increasing correspondences defined on posets more general than lattices. An interesting class of posets suggesting possible approaches to this problem is that of Noetherian posets. A poset $X$ is Noetherian if every nonempty subset of $X$ has a maximal element. Birkhoff (1967, Chapter VIII) provieds for an account of Noetherian poset. A poset $X$ is a complete lattice if and only if it is a Noetherian lattice with a least element. The following fact makes Noetherian posets a good starting point to study fixpoints of upper increasing correspondences defined on non-lattice posets.

Theorem 1.1: Let $X$ be a Noetherian poset and $F: X \rightarrow X$ be a nonempty, upper increasing correspondence. Every maximal element of $A_{F}$ is a fixpoint of $F$.

Proof: Take any maximal element $m$ of $A_{F}$. Some such element exists because $A_{F}$ is nonempty and $X$ is Noetherian. Since $m \in A_{F}$, there exists some $y \in F_{m}$ such that $m \leq y$. Hence, by upper increasingness of $F$, there exists some $z \in F_{y}$ such that $y \leq z$. Thus $y \in A_{F}$, and since $m$ is maximal for $A_{F}, m \leq y$ implies that $m=y$. Hence $m$ is a fixpoint of $F$. Q.E.D

Theorem 1.2 below, using the Axiom of Choice, provides for sufficient conditions for $A_{F}$ to have a maximal element. d'Orey (1996) presents results in the same vein, but using a notion of increasingness for $F$ much strongher than upper increasingness. Theorem 1.2 can easily be turned into a generalization of Theorem 2 in d'Orey (1996). 
Theorem 1.2: Let $X$ be a chain complete poset and and $F: X \rightarrow X$ be a nonempty, upper increasing correspondence such that $F_{x}$ has a greatest element for every $x \in X$. Then $F$ has a fixpoint.

Proof: We simply redo the argument in the first part of the proof of Theorem 1, and then apply Theorem 1.1. Let $C$ be any nonempty chain of $A_{F}$. Let $c^{*}$ be the sup of $C$. Pick any $x \in C$. Since $C \subseteq A_{F}$, then by the definition of $A_{F}$ there exists $y_{x} \in F_{x}$ such that $x \leq y_{x}$. Since $x \leq c^{*}$, by upper increasingness of $F$ there exists $z_{x} \in F_{c^{*}}$ such that $y_{x} \leq z_{x}$. Hence, for the greatest element $1_{c^{*}}$ of $F_{c^{*}}, x \leq y_{x} \leq z_{x} \leq 1_{c^{*}}$. Since $1_{c^{*}}$ does not depend on the chosen $x$, then for every $x \in C, x \leq 1_{c^{*}}$. Thus $1_{c^{*}}$ majorizes $C$ and so $c^{*} \leq 1_{c^{*}}$. Hence $c^{*} \in A_{F}$ since $1_{c^{*}} \in F_{c^{*}}$. Because $C$ was arbitrary, every nonempty chain of $A_{F}$ has a supremun in $A_{F}$. Thus, by Zorn's Lemma, $A_{F}$ has a maximal element. By Theorem 1.1, such a maximal element is a fixpoint of F. Q.E.D

Theorem 2 below generalizes the "structure" part of Zhou's fixpoint theorem (1994, Theorem 1, point (iii)) in two repects. First, it requires $F$ to be upper increasing and strongly lower increasing, instead of asking it to be Veinott increasing (assumption (a)).

Second, Theorem 2 requires $F_{x}$ to have greatest and least elements for every $x \in X$ (assumption (b)) and it requires, for every $a \in X$ at wich $G^{a}$ is well definded (i.e. $U^{a}$ is nonempty) and for every $x \in U^{a}$, that the set $G_{x}^{a}=F_{x} \cap[a, 1]$ has a least element (assumption (c)). We need assumption (c) because the least element of $F_{x}$ is not necessarily retained in $G_{x}^{a}$. Zhou's theorem assumes on the other hand that $F_{x}$ is a subcomplete sublattice of $X$ for every $x \in X$. To see that Zhou's assumption is strongher, note that, whenever $F_{x}$ is a subcomplete sublattice of $X$, the set $G_{x}^{a}=F_{x} \cap[a, 1]$, if nonempty, is a nonempty subcomplete sublattice of $X$, being the intersection of two nonempty subcomplete sublattices of $X$, and so has a least (and a greatest) element.

The property of $G_{x}^{a}=F_{x} \cap[a, 1]$ having a least element if nonempty would be implied also by assuming that $F_{x}$ is a complete lattice, instead of 
a subcomplete sublattice of $X$. Indeed, while in general the intersection of a lattice which is a subset of $X$ with a subcomplete sublattice of $X$ needs not to be a subcomplete sublattice of $X$, it is so when the subcomplete sublattice has the form of a closed interval $[y, 1]$. This can be proved very easily.

Theorem 2: Let $X$ be a complete lattice and $F: X \rightarrow X$ be a nonempty correspondence. If (a) $F$ is upper increasing and strongly lower increasing, (b) for every $x \in X, F_{x}$ has a greates element and a least element, and (c) for every $a \in X$ such that $U^{a} \neq \emptyset$ and for every $x \in U^{a}, G_{x}^{a}$ has a least element, then the fixpoint set of $F$ is a nonempty complete lattice.

Proof: From Theorem 1, point (i), $E_{F}$ is nonempty. Take any nonempty subset $H \subset E_{F}$. Since $X$ is join-complete, $h^{*}:=\sup H$ does exist.

We want to prove that $\sup _{E_{F}} H$ does exist. This is equivalent to proving that the intersection $E_{F} \cap\left[h^{*}, 1\right]$ has a least element, where 1 is the greatest element of $X$. We follow Tarski's argument.

We first show that $h^{*} \in A_{F}$. This is the same argument as that in the first part of the proof of Theorem 1. Take any $x \in H$. Since $H \subseteq E_{F} \subseteq A_{F}$, there exists $y_{x} \in F_{x}$ such that $x \leq y_{x}$. Furthermore, since $x \leq h^{*}$ then by upper increasingness of $F$, for any such $y_{x} \in F_{x}$ there exists $z_{x} \in F_{h^{*}}$ such that $y_{x} \leq z_{x}$. Hence, for the greatest element $1_{h^{*}}$ of $F_{h^{*}}, x \leq y_{x} \leq z_{x} \leq 1_{h^{*}}$. Thus $1_{h^{*}}$ majorizes $H$ because it does not depend on the chosen $x$, and so $h^{*} \leq 1_{h^{*}}$. Since $1_{h^{*}} \in F_{h^{*}}$, we are done.

$h^{*} \in A_{F}$ implies that $h^{*} \in U^{h^{*}}$. We now show that $\left(h^{*}, 1\right] \subseteq U^{h^{*}}$, hence that the restriction $\Gamma^{h^{*}}$ of the correspondence $G^{h^{*}}: U^{h^{*}} \rightarrow\left[h^{*}, 1\right]$ to the interval $\left[h^{*}, 1\right]$ is well defined. This amount to show that for every $x \in X$ such that $h^{*}<x, F_{x} \cap\left[h^{*}, 1\right] \neq \emptyset$. Take indeed any $x \in X$ such that $h^{*}<x$. Since $h^{*} \in A_{F}$, there exists some $y \in F_{h^{*}}$ such that $h^{*} \leq y$. By upper increasingness of $F, h^{*}<x$ implies that for any such $y \in F_{h^{*}}$ there exists $z \in F_{x}$ such that $y \leq z$. Hence $h^{*} \leq y \leq z$, and we are done.

The restriction $\Gamma^{h^{*}}$ is thus a correspondence mapping elements of the complete lattice $\left[h^{*}, 1\right]$ into nonempty subsets of $\left[h^{*}, 1\right]$. By assumption $G^{h^{*}}$ is lower increasing, and so $\Gamma^{h^{*}}$ is lower increasing. Furthermore, by assumption 
$G_{x}^{h^{*}}$ has a least element for every $x \in U^{h^{*}}$. Hence $\Gamma_{x}^{h^{*}}$ has a least element for every $x \in\left[h^{*}, 1\right]$. Thus, by point (ii) of Theorem $2, \Gamma^{h^{*}}$ has a least fixpoint. Because by construction the fixpoint set of $\Gamma^{h^{*}}$ is exactly $E_{F} \cap\left[h^{*}, 1\right]$, then $H$ has a supremum in $E_{F}$.

Since $H$ has been taken arbitrarily, then $E_{F}$ is a nonempty join-complete lattice. Because $F$ is strongly lower increasing, then it is lower increasing, and since $F_{x}$ has a least element for every $x \in X$, then by point (ii) of Theorem $1, E_{F}$ has a least element. Hence $E_{F}$ is a nonempty complete lattice. Q.E.D

\section{Games with complementarities}

A game in normalized form is a pair $\left(I,\left(X^{i}, u^{i}\right)_{i \in I}\right)$; where $I$ is a nonempty and finite set of players and for every $i \in I, X^{i}$ is player $i$ 's nonempty strategy set and $u^{i}: X \rightarrow \mathbb{R}$ is $i$ 's payoff function, with $X:=\prod_{i \in I} X^{i}$.

Let $T^{i}:=\prod_{j \in I, j \neq i} X^{j}$, the set of $i$ 's opponents strategy profiles.

Player $i$ 's best reply correspondence is:

$$
B^{i}: t^{i} \in T^{i} \mapsto B_{t^{i}}^{i}:=\operatorname{argmax}_{x^{i} \in X^{i}} u^{i}\left(x^{i}, t^{i}\right) \subseteq X^{i}
$$

The joint best reply correspondece is:

$$
B: x \in X \mapsto B_{x}:=\prod_{i \in I} B_{t^{i}}^{i} \subseteq X
$$

The nash equilibria of the game are the fixpoints of the joint best reply $B$.

We could apply the theorems of the previous section to games in normal form on setting $F=B$ and, for any $a=\left(a^{1}, \ldots, a^{I}\right) \in X, G_{x}^{a}=$ $B_{x} \cap[a, 1]$, the right-hand-side being equal to $\left\{\prod_{i \in I} B_{t^{i}}^{i}\right\} \cap\left\{\prod_{i \in I}\left[a_{i}, 1_{i}\right]\right\}=$ $\prod_{i \in I}\left\{B_{t^{i}}^{i} \cap\left[a_{i}, 1_{i}\right]\right\}$.

For a given reference-point of strategies $a=\left(a^{1}, \ldots, a^{I}\right) \in X$, the set $G_{x}^{a}$ 
if nonempty is the set of strategy profiles $y$ which are both best against $x$ and greater than the reference profile $a$. Since $X$ has a product order, it means that every entry $y_{i}$ in such a $y$, is both best against $\left(x^{1}, \ldots, x^{i-1}, \ldots, x^{i+1}, \ldots, x^{I}\right)$ and such that $a_{i} \leq y_{i}$.

We define now our class of games with complementarities. The definition is refereed to the normal form, but it applies to trees upon considering the associated reduced normal form.

Definition 3 (games with complementarities): Let $X$ be a complete lattice. A game has complementarities if its joint best reply $B$ is nonempty, if it is increasing according to Definition 1 , and if $B_{x}$ has a least and a greatest element for every strategy profile $x \in X$. A game has strong complementarities if it has complementarities and, furthermore, if its joint best reply is strongly lower increasing according to Definition 2 and $G_{x}^{a}$ has a least elements whenever nonempty.

By Theorem 1, a game with complementarities has a least and a greatest Nash equilibrium. By Theorem 2, the Nash set of a game with strong complementarities is a nonempty complete lattice.

Relation with the existing literature. In the literature, the widest known class of games with complementarities is the class where, for each game, $X$ is a complete lattice, the joint best reply $B$ is nonempty and is at least Veinott-increasing, and the best response sets $B_{x}$ are subcomplete sublattices of $X$ for every profile $x \in X$. These games, that we call Veinott games, are studied in Milgrom and Shannon (1994), Topkis (1979) and Vives (1990). The latter is an instance where the monotonicity notion used for the best replies is strongher than Veinott increasingness. The main property of Veinott games is that, by Zhou's fixpoint theorem, they have a Nash set which is a nonempty complete lattice. As we have seen in Section 3, every Veinott game is a game with strong strategic complementarities, according to Definition 3. We have also shown in the examples of Section 3 that the 
converse is false ${ }^{3}$. Hence our games with strong complementarities have the Veinott games as a strict subset, and so our games with complementarities, too, have the Veinott games as a strict subset.

A bounch of work has been devoted to find sufficient conditions on the payoffs guaranteeing that a game is a Veinott game. In terms of sufficient condition on payoffs, the widest class of Veinott games known in the literature is that of quasisupermodular games. These games are introduced by Milgrom and Shannon (1994), and they have as special cases the well-known supermodular games of Topkis (1979, Section 3) and Vives (1990, Theorem $4.2)$.

Our goal in this section is to find sufficient condition on the payoffs guaranteeing that a game has complementarities accordingly to Definition 3. Not surprisingly, these conditions turn out to be weaker than those in quasisupermodular games. Hence, we can define by means of properties on payoffs a subclass of games with complementarities which is strictly wider than that of quasisupermodular games of Milgrom and Shannon and, of course, of that of supermodular games of Topkis and Vives.

We start in subsection 4.1 with a recap of quasisupermodular games, to allow comparison. In section 4.2 we present our conditions on the payoffs and the monotone comparative statices they generate. In subsection 4.3 we extend the analysis to games.

\subsection{Recap of quasisupermodular games}

Milgrom and Shannon (1994) introduced the class of quasisupermodular games. This class includes the supermodular games of Topkis (1979) and Vives (1990) as a special case.

Quasisupermodular games are games whose payoffs are quasisupermodular and satisfy a single crossing condition. These properties express, respectively, a weak form of complementarity among own strategies and among

\footnotetext{
${ }^{3}$ All this holds even if one required, in Veinott games, that sets $B_{x}$ were only complete lattices, not necessarily subcomplete sublattices of $X$.
} 
own strategies and opponents' profiles. The relevance of qasisupermodularity and the single crossing condition is that they suffice to make the joint best reply Veinott-increasing, albeit they are not necessary, and to make the best reponse sets sublattices of the strategy spaces.

Quasisupermodularity is an ordinal generlization of supermodularity. The single crossing condition is an ordinal generalization of the condition of increasing differences. Topkis (1998) explores this further. We report here the definitions. Let $X^{i}$ be a lattice and $T^{i}$ be a poset.

Payoff $u^{i}\left(x^{i}, t^{i}\right)$ is quasisupermodular in $x^{i}$ on $X^{i}$ if $\forall z_{1}, z_{2} \in X^{i}, \forall t \in T^{i}$,

$$
\begin{aligned}
& u^{i}\left(\inf \left\{z_{1}, z_{2}\right\}, t\right) \leq u^{i}\left(z_{1}, t\right) \Rightarrow u^{i}\left(z_{2}, t\right) \leq u^{i}\left(\sup \left\{z_{1}, z_{2}\right\}, t\right) ; \\
& u^{i}\left(\sup \left\{z_{1}, z_{2}\right\}, t\right) \leq u^{i}\left(z_{2}, t_{i}\right) \Rightarrow u^{i}\left(z_{1}, t\right) \leq u^{i}\left(\inf \left\{z_{1}, z_{2}\right\}, t\right) .
\end{aligned}
$$

Payoff $u^{i}\left(x^{i}, t^{i}\right)$ satisfies the single crossing property in $\left(x^{i}, t^{i}\right)$ on $X^{i} \times T^{i}$ if $\forall y_{1}, y_{2} \in X^{i}$ such that $y_{1} \leq y_{2}, \forall t_{1}, t_{2} \in T^{i}$ such that $t_{1} \leq t_{2}$,

$$
\begin{aligned}
& u^{i}\left(y_{1}, t_{1}\right) \leq u^{i}\left(y_{2}, t_{1}\right) \Rightarrow u^{i}\left(y_{1}, t_{2}\right) \leq u^{i}\left(y_{2}, t_{2}\right) \\
& u^{i}\left(y_{2}, t_{2}\right) \leq u^{i}\left(y_{1}, t_{2}\right) \Rightarrow u^{i}\left(y_{2}, t_{1}\right) \leq u^{i}\left(y_{1}, t_{1}\right) .
\end{aligned}
$$

A game in normal form is quasisupermodular if for every player $i$, the payoff $u^{i}\left(x^{i}, t^{i}\right)$ is quasisupermodular on $X^{i}$, and satisfies the single crossing property on $X^{i} \times T^{i}$. Furthermore, to make the individual best replies nonempty, it is assumed that the strategy set $X^{i}$ is a lattice compat in any topology finer than its interval topology, and the payoff is upper semicontinuous on $X^{i}$ for every $t \in T^{i}$.

For quasisupermodular games, the monotone comparative statics of the individual best replies makes it possible to apply Zhou's fixpoint theorem to show that the Nash set is a nonempty complete lattice.

In particular, Milgrom and Shannon (1994, Th. 4) shows that each individual best reply is Veinott-increasing as opponents' profile increases. As a special case, each such best reply takes values in the collection of sublattices of own strategies. If now own strategy sets are complete lattices, and payoff 
are semicontinuos in own strategies, then each individual best reply is also compact-valued, and being a sublattice it is a subcomplete sublattice of the set of own strategies by a theorem of Birkhoff (see Topkis, 1998, for a refined result in finite euclidean spaces). All this is preserved under finite cartesian products, and so Zhou's fixpoint theorem applies and the Nash set of the game is a nonempty complete lattice.

\subsection{Monotone comparative statics over directed posets}

We introduce here conditions on payoffs weaker that quasisupermodularity and the single crossing, and making the solution correpondences increasing in the sense of Definition 1. This section is devoted to monotone comparative statics of individual optimization problems, and as such has a wider scope than its application to games. We maintain the notation of our game setting for convenience.

Our order-theoretic notion in this section is that of a directed poset. A partially ordered set $X$ is downward directed if any two elements $x, y \in X$ have an lower bound in $X$. It is upward directed if any two elements $x, y \in X$ have and upper bound in $X$. It is directed if it is both downward and upward direcred. Clearly a lattice is a directed poset, while the converse is false. A consumer competitive budget set is downward directed, the common lower bound of all the pairs of consumption bundles being the no-consumption vector (the origin).

We now introduce properties on payoffs wich are weaker than quasisupermodularity plus the single crossing, while still expressing a form of complementarity ${ }^{4}$.

Definition 4 (upper modular functions): Let $X^{i}$ be a downward directed poset and $T^{i}$ be a poset. Payoff $u^{i}\left(x^{i}, t^{i}\right)$ is upper modular if for every $x_{1}, x_{2} \in X^{i}$ such that $x_{1} \not \leq x_{2}$ there exist elements $a_{x_{1} x_{2}}, b_{x_{1} x_{2}} \in X^{i}$,

\footnotetext{
${ }^{4}$ Suggestions for names better than those in the following definitions are welcomed.
} 
with $a<x_{1} \leq b$, such that for every $t \in T^{i}$,

$$
u^{i}(a, t) \leq u^{i}\left(x_{1}, t\right) \Rightarrow u^{i}\left(x_{2}, t\right) \leq u^{i}(b, t)
$$

and for every $t_{1}, t_{2} \in T^{i}$ such that $t_{1} \leq t_{2}$,

$$
u^{i}\left(a, t_{1}\right) \leq u^{i}\left(x_{1}, t_{1}\right) \Rightarrow u^{i}\left(a, t_{2}\right) \leq u^{i}\left(x_{1}, t_{2}\right) .
$$

Remark. Points $a$ and $b$ depends on both $x_{1}$ and $x_{2}$. It is to remark this, that we explicitly indexed $a$ and $b$ with $x_{1}, x_{2}$ in the beginning of the definition. We removed the indexing in the rest of the definition and in proofs below to easy notation. If $x_{2}<x_{1}$, we know that some $a$ in $X^{i}$ with $a<x_{1}$ do exists, namely $x_{2}$. If, on the other hand, $x_{1}, x_{2}$ are unordered, we know that some $a, b \in X^{i}$ such that $a<x_{1} \leq b$ do exist. For example, any lower bound of $x_{1}, x_{2}$ will work as $a$, such lower bound existing because $X^{i}$ is donward directed. Of course $b$ can be $x_{1}$ itself. An analogous remark applies to the following definition.

Definition 5 (lower modular functions): Let $X^{i}$ be an upward directed poset and $T^{i}$ be a poset. Payoff $u^{i}\left(x^{i}, t^{i}\right)$ is lower modular if for every $x_{1}, x_{2} \in X^{i}$ such that $x_{1} \not \leq x_{2}$ there exist elements $c_{x_{1} x_{2}}, d_{x_{1} x_{2}} \in X^{i}$, with $c \leq x_{2}<d$, such that for every $t \in T^{i}$,

$$
u^{i}(d, t) \leq u^{i}\left(x_{2}, t\right) \Rightarrow u^{i}\left(x_{1}, t\right) \leq u^{i}(c, t)
$$

and for every $t_{1}, t_{2} \in T^{i}$ such that $t_{1} \leq t_{2}$,

$$
u^{i}\left(d, t_{2}\right) \leq u^{i}\left(x_{2}, t_{2}\right) \Rightarrow u^{i}\left(d, t_{1}\right) \leq u^{i}\left(x_{2}, t_{1}\right) .
$$

That upper and lower modularity follows from quasisupermodularity and 
the single crossing property id obvious. Let $X^{i}$ be a lattice and pick $x_{1}, x_{2} \in$ $X^{i}$ such that $x_{1} \not \leq x_{2}$. Set $a_{x_{1} x_{2}}=\inf \left\{x_{1}, x_{2}\right\}$. Note that $a<x_{1}$. Assume the hypothesis in (7) holds. By $(3), u^{i}\left(x_{2}, t\right) \leq u^{i}\left(\sup \left\{x_{1}, x_{2}\right\}, t\right)$. Hence (7) holds on setting $b_{x_{1} x_{2}}=\sup \left\{x_{1}, x_{2}\right\}$, and seeing that $b=x_{1}$ if $x_{2}<x_{1}$ and $x_{1}<b$ if $x_{1}, x_{2}$ are unordered. The same reasonoing shows that (8) comes from (5), because $a_{x_{1} x_{2}}<x_{1}$. Analogously, lower modularity follows from (4) and (6) with $c_{x_{1} x_{2}}=\inf \left\{x_{1}, x_{2}\right\}$ and $d_{x_{1} x_{2}}=\sup \left\{x_{1}, x_{2}\right\}$.

The difference between upper modularity and quasisupermodularity is that in the former, $a$ is not required to be the inf and $b$ must be greater than $x_{1}$ only. Analogously for lower modularity.

Example 4: A function which is upper modular but not quasisupermodular. Take the lattice $X^{i}=\left\{x_{1}, x_{2}\right.$, inf $\left\{x_{1}, x_{2}\right\}$, sup $\left.\left\{x_{1}, x_{2}\right\}\right\}$, with $x_{1}, x_{2}$ unordered. Consider the function $u^{i}\left(x_{1}\right)=4, u^{i}\left(x_{2}\right)=2$, $u^{i}\left(\inf \left\{x_{1}, x_{2}\right\}\right)=3, u^{i}\left(\sup \left\{x_{1}, x_{2}\right\}\right)=1$. Quasisupermodularity fails at the pair of points $\left(x_{1}, x_{2}\right)$ and holds vacuously at the pair of points $\left(x_{2}, x_{1}\right)$. Upper modularity holds at $\left(x_{1}, x_{2}\right)$, on setting $a=\inf \left\{x_{1}, x_{2}\right\}$ and $b=x_{1}$, and holds vacuously at $\left(x_{2}, x_{1}\right)$. The morale in this example is the possibility to set $b=x_{1}$ in the definition of upper modularity.

The following lemma describes the comparative statics of individual best replies generated by the payoff being upper and lower modular.

Lemma 1: Let $X^{i}$ be a downward directed poset and $T^{i}$ be a poset. If $u^{i}: X^{i} \times T^{i} \rightarrow \mathbb{R}$ is upper modular, then

$$
B^{i}: t^{i} \in T^{i} \mapsto B_{t^{i}}^{i}=\operatorname{argmax}_{x^{i} \in X^{i}} u^{i}\left(x^{i}, t^{i}\right)
$$

is upper increasing, if nonempty. Let $X^{i}$ be an upward directed poset and $T^{i}$ be a poset. If $u^{i}$ is lower modular, then $B^{i}$ is lower increasing if nonempty.

Proof: Take any two profiles $t_{1}, t_{2} \in T^{i}$ such that $t_{1} \leq t_{2}$. The proof amounts to properly relabeling the proof of the Monotonicity Theorem of Milgrom and Shannon (1994, Th.4). We start with upper increasingness. 
Pick any $s \in B_{t_{1}}^{i}$ and any $x_{2} \in B_{t_{2}}^{i}$. If $s \leq x_{2}$ we are done. So suppose $s \not \leq x_{2}$. Since $s$ is best against $t_{1}$, for every $x \in X^{i}$ we have $u^{i}\left(x, t_{1}\right) \leq u^{i}\left(s, t_{1}\right)$. Hence for $a_{s, x_{2}} \in X^{i}, u^{i}\left(a, t_{1}\right) \leq u^{i}\left(s, t_{1}\right)$. By (8), $u^{i}\left(a, t_{2}\right) \leq u^{i}\left(s, t_{2}\right)$, and there exists some $b_{s, x_{2}} \in X^{i}$, with $s \leq b$, such that according to (7), $u^{i}\left(x_{2}, t_{2}\right) \leq u^{i}\left(b, t_{2}\right)$. Hence $b \in B_{t_{2}}^{i}$ because $x_{2}$ is optimal against $t_{2}$. And we are done.

We now show that $B^{i}$ is lower increasing. Take any $v \in B_{t_{2}}^{i}$ and any $x_{1} \in B_{t_{1}}^{i}$. For every $x \in X^{i} u^{i}\left(x, t_{2}\right) \leq u^{i}\left(v, t_{2}\right)$. Hence for $d_{x_{1}, v} \in X^{i}$, $u^{i}\left(d, t_{2}\right) \leq u^{i}\left(v, t_{2}\right)$. By $(10), u^{i}\left(d, t_{1}\right) \leq u^{i}\left(v, t_{1}\right)$, and there exists some $c_{x_{1}, v} \in X^{i}$, with $c \leq v$, such that according to (9), $u^{i}\left(x_{1}, t_{1}\right) \leq u^{i}\left(c, t_{1}\right)$. Hence $c \in B_{t_{1}}^{i}$ because $x_{1}$ is best against $t_{1}$, and we are done. Q.E.D

Our monotone comparative statics says nothing about the structure of the sets $B_{t}^{i}$. If we strengthen upper modularity to strong upper modulariy, as stated in Definition 6 below, we obtain that every $B_{t^{i}}^{i}$ is a chain and that $B^{i}$ satisfies a form of increasingness strongher than Veinott increasingness.

In Definition 6 we strengthen upper modularity by distinguishing, for every $x_{1}, x_{2} \in X^{i}$ such that $x_{1} \not \leq x_{2}$, the case where $x_{1}$ and $x_{2}$ are unordered from the case where $x_{2}<x_{1}$. In the first case, the generalizaed crossing condition (8) holds as such, and (7) is strengthened to (11). In the second case, the generalized quasisupermodularity (7) holds as such, (12) is added, and (8) is strengthened to (13).

The notion of strong upper modulariy in Definition 6 is weaker than strong quasisupermodularity plus strong single crossing property, as these latter are defined in Shannon (1995). To see this just set, for every $x_{1}, x_{2} \in X^{i}$ such that $x_{1} \not \leq x_{2}, a_{x_{1} x_{2}}=\inf \left\{x_{1}, x_{2}\right\}$ and $b_{x_{1} x_{2}}=\sup \left\{x_{1}, x_{2}\right\}$. Then (11) follows from strict quasisupermodulariy, (12) from quasisupermodulary, and (13) from the strict single crossing property.

Lemma 2 is a generalization of the sufficiency part of Shannon (1995)'s theorem 4 in the case where the constraint set stays put. Indeed, that theorem holds already for payoffs that are strictly quasisupermodular at unordered $x_{1}, x_{2}$, and that satisty the strict single crossing property at pairs 
$\left(x_{1}, x_{2}\right)$ such that $x_{2}<x_{1}$.

Definition 6 (strongly upper modular functions): Let $X^{i}$ be a lower directed poset and $T^{i}$ be a poset. Payoff $u^{i}\left(x^{i}, t^{i}\right)$ is strongly upper modular if it is upper modular and, for every $x_{1}, x_{2} \in X^{i}$ such that $x_{1} \not \leq x_{2}$ :

(a) if $x_{1}, x_{2}$ are unordered then for every $t \in T^{i}$,

$$
u^{i}(a, t) \leq u^{i}\left(x_{1}, t\right) \Rightarrow u^{i}\left(x_{2}, t\right)<u^{i}(b, t)
$$

(b) if $x_{2}<x_{1}$ then, for every $t \in T^{i}$,

$$
u^{i}(a, t)<u^{i}\left(x_{1}, t\right) \Rightarrow u^{i}\left(x_{2}, t\right)<u^{i}(b, t)
$$

and for every $t_{1}, t_{2} \in T^{i}$ with $t_{1}<t_{2}$,

$$
u^{i}\left(a, t_{1}\right) \leq u^{i}\left(x_{1}, t_{1}\right) \Rightarrow u^{i}\left(a, t_{2}\right)<u^{i}\left(x_{1}, t_{2}\right) .
$$

Lemma 2: Let $X^{i}$ be a downward directed poset and $T^{i}$ be a poset. If $u^{i}: X^{i} \times T^{i} \rightarrow \mathbb{R}$ is strongly upper modular, then for every $t_{1}, t_{2} \in T^{i}$ such that $t_{1}<t_{2}$, for every $s \in B_{t_{1}}^{i}$ and every $v \in B_{t_{2}}^{i}, s \leq v$. Furthermore, for every $t \in T^{i}, B_{t}^{i}$ is a chain.

Proof: Take any two profiles $t_{1}, t_{2} \in T^{i}$ such that $t_{1}<t_{2}$. Pick any $s \in B_{t_{1}}^{i}$ and any $v \in B_{t_{2}}^{i}$. Suppose $s, v$ are unordered. Since $s$ is best against $t_{1}$, for every $x \in X^{i}$ it holds that $u^{i}\left(x, t_{1}\right) \leq u^{i}\left(s, t_{1}\right)$. Hence for $a_{s, v} \in X^{i}$, $u^{i}\left(a, t_{1}\right) \leq u^{i}\left(s, t_{1}\right)$. By (8), $u^{i}\left(a, t_{2}\right) \leq u^{i}\left(s, t_{2}\right)$, and so there exists some $b_{s, v} \in X^{i}$, with $s \leq b$, such that according to (11) $u^{i}\left(v, t_{2}\right)<u^{i}\left(b, t_{2}\right)$, a contradiction. Hence $s$ and $v$ must be ordered. Suppose now that $v<s$. Then by (13) (with $x_{1}=s$ ), for $a_{s, v} \in X^{i}$, since $u^{i}\left(a, t_{1}\right) \leq u^{i}\left(s, t_{1}\right)$ then $u^{i}\left(a, t_{2}\right)<u^{i}\left(s, t_{2}\right)$. Hence by (12) (with $x_{2}=v$ ), for $b_{s, v} \in X^{i}, u^{i}\left(v, t_{2}\right)<$ $u^{i}\left(b, t_{2}\right)$, a contradiction. Hence $s \leq v$.

Take now $s, v \in B_{t}^{i}$, for any $t \in T^{i}$. If they are unordered, we can use 
(11) to reach a contradiction. Hence $B_{t}^{i}$ is a chain. Q.E.D

To say something on the structure of the sets $B_{t}^{i}$ without generating a too strong monotonicity of the solution correspondece $B^{i}$, we introduce now a property on payoffs which is still weaker than quasisupermodularity plus single crossing, but strongher than upper and lower modularity. A cardinal version of the property has been introduced by d'Orey (1996, Definition 5).

Definition 7 (bound-modular functions): Let $X^{i}$ be a directed poset and $T^{i}$ be a poset. Payoff $u^{i}\left(x^{i}, t^{i}\right)$ is bound- modular if for every $x_{1}, x_{2} \in X^{i}$, there exist in $X^{i}$ an upper bound $U$ and a lower bound $L$ of $\left\{x_{1}, x_{2}\right\}$ such that for every $t \in T^{i}$,

$$
\begin{aligned}
& u^{i}(L, t) \leq u^{i}\left(x_{1}, t\right) \Rightarrow u^{i}\left(x_{2}, t\right) \leq u^{i}(U, t) \\
& u^{i}(U, t) \leq u^{i}\left(x_{2}, t\right) \Rightarrow u^{i}\left(x_{1}, t\right) \leq u^{i}(L, t) ;
\end{aligned}
$$

and for every $t_{1}, t_{2} \in T^{i}$ such that $t_{1} \leq t_{2}$,

$$
\begin{aligned}
& u^{i}\left(L, t_{1}\right) \leq u^{i}\left(x_{1}, t_{1}\right) \Rightarrow u^{i}\left(L, t_{2}\right) \leq u^{i}\left(x_{1}, t_{2}\right) \\
& u^{i}\left(U, t_{2}\right) \leq u^{i}\left(x_{2}, t_{2}\right) \Rightarrow u^{i}\left(U, t_{1}\right) \leq u^{i}\left(x_{2}, t_{1}\right)
\end{aligned}
$$

The proof of the following Lemma is immediate from theorem 4 in Milgrom and Shannon (1994), and is omitted. Note that the monotonicity of the solution correspondece that we generate by bound-modularity is strongher than upper and lower increasingness.

Lemma 3: Let $X^{i}$ be a directed poset and $T^{i}$ be a poset. If $u^{i}: X^{i} \times T^{i} \rightarrow$ $\mathbb{R}$ is bound-modular, then for every $t_{1}, t_{2} \in T^{i}$ such that $t_{1} \leq t_{2}$, for every $x_{1} \in B_{t_{1}}^{i}$ and every $x_{2} \in B_{t_{2}}^{i}$, there are in $X^{i}$ a lower bound $L$ and an upper bound $U$ of $\left\{x_{1}, x_{2}\right\}$ such that $L \in B_{t_{1}}^{i}$ and $U \in B_{t_{2}}^{i}$. Furthermore, for every $t \in T^{i}, B_{t}^{i}$ is a directed poset. 


\subsection{Classes of games with complementarities}

The following theorem defines a subclass of games with strong complementarities. This subclass has the strictly quasisupermodular games, i.e. the games where payoffs are striclty quasisupermodular and satisfy the strict single crossing property, as special cases. For example the strictly supermodular games studied by Vives (1990, Theorem 4.2) are special cases of the games defined and studied in Theorem 3.

In the proof of Theorem 3 we will use Lemma 2. We point out that increasingness as in Definition 1, and strong lower increasingness as in Definition 2, are preserved under cartesian products, contrary to the increasingness notion used for example in Vives (1990) and d'Orey (1996), see Remark 4.1 in Vives (1990). This is why we can prove Theorem 3.

Theorem 3: Let $\left(I,\left(X^{i}, u^{i}\right)_{i \in I}\right)$ be a game in normal form where, for every player $i, X^{i}$ is a lattice compact in any topology finer than its interval topology and payoff $u^{i}: X^{i} \times T^{i} \rightarrow \mathbb{R}$ is upper semicontinuous in own strategies, lower modular and strongly upper modular. Then the game has strong complementarities, and so its Nash set is a nonempty complete lattice.

Proof: Fix any player $i \in I$. Since $X^{i}$ is compact and $u^{i}$ is upper semicontinuos, then $B^{i}$ is nonempty and compact. By Lemma 2 , for every $t^{i} \in T^{i}$, $B_{t^{i}}^{i}$ is a chain, and being compact it has a least and a greatest element. Furthermore, by Lemma $1, B^{i}$ is both upper and lower increasing (Definition 1). Is is immediate to see that upper and lower increasingness of $B^{i}$, for every $i \in I$, implies that $B=\Pi_{i} B^{i}$ is both upper and lower increasing. Furthermore, since every $X^{i}$ is a complete lattice then $X=\Pi_{i} X^{i}$ is a complete lattice. Finally, $B$ has a least and a greatest element because every $B^{i}$ has a least and a greatest element. Thus the game has complementarities according to Definition 3. So, by Theorem 1, its Nash set has a least and a greatest element.

Fix now a profile of strategies $a \in X$ such that $U^{a}$ is nonempty. Consider the correspondence $G^{a}: x \in U^{a} \mapsto G_{x}^{a}=B_{x} \cap[a, 1]$. Note that for every $x \in$ 
$U^{a}, G_{x}^{a}=\Pi_{i} B_{t^{i}}^{i} \cap\left[a^{i}, 1^{i}\right]$ where $1^{i}$ is the greatest element of $X^{i}$. Hence every $a \in X$ with $U^{a} \neq \emptyset$ define $\operatorname{card}(I)$ nonempty correspondences $t^{i} \in U_{a^{i}}^{a} \mapsto$ $B_{t^{i}}^{i} \cap\left[a^{i}, 1^{i}\right]$, where $U_{a^{i}}^{a}=\left\{t^{i} \in T^{i}:\left(a^{i}, t^{i}\right) \in U^{a}\right\}$ is the section of $U^{a}$ at $a^{i}$ given $i \in I$. For every $i \in I$, by Lemma 2 the correspondence $t^{i} \in U_{a^{i}}^{a} \mapsto B_{t^{i}}^{i}$ is Veinott increasing. Hence the correspondence $t^{i} \in U_{a^{i}}^{a} \mapsto B_{t^{i}}^{i} \cap\left[a^{i}, 1^{i}\right]$ is also Veinott increasing, and so it is lower increasing. Thus the product $G_{x}^{a}$ is lower increasing on $U^{a}$, and since $a$ was arbitrary, $B$ is strongly lower increasing.

Furthermore, by Lemma 2, for every $t^{i} \in T^{i}, B_{t^{i}}^{i}$ is a compact chain. So for every $t^{i} \in U_{a^{i}}^{a}, B_{t^{i}}^{i} \cap\left[a^{i}, 1^{i}\right]$ is also a compact chain, and as such it has a least element. Hence for every $a \in X$ with $U^{a} \neq \emptyset, G_{x}^{a}$ has a least element.

Thus, according to Definition 3, the game has strong complementarities, and by Theorem 2 its Nash set is a nonempty complete lattice. Q.E.D

\section{REFERENCES}

Birkhoff, G., 1967. Lattice Theory. Third edition, AMC Colloquium Publications, volume XXV.

d'Orey, V., 1996. Fixed point theorems for correspondences with values in a partially ordered set and extended supermodular games. Journal of Mathematical Economics. 25, 345-354.

Milgrom, P. and C. Shannon, 1994. Monotone comparative statics. Econometrica.

Samuelson, P.A, 1974. Complementarity - an essay on the 40th anniversary of the Hicks-Allen revolution in demand theory. Journal of Economic Literature. 2, 1255-1289.

Shannon, C., 1995. Weak and strong comparative statics. Economic Theory. 5, 209-227.

Tarski, A., 1955. A lattice-theoretical fixpoint theorem and its applications. Pacific Journal of Mathematics. 5, 285-309.

Topkis, D.M., 1978. Minimizing a submodular function on a lattice. Op- 
erations Research. 26, 305-321.

Topkis, D.M., 1979. Equilibrium points in nonzero-sum n-person submodular games. SIAM Journal on Control and Optimization. 17, 773-787.

Topkis, D.M., 1998. Supermodularity and complementarity. Princeton University Press.

Vives, X., 1990. Nash equilibrium with strategic complementarities. Journal of Mathematical Economics. 19, 305-321.

Zhou, L., 1994. The set of Nash equilibria of a supermodular game is a complete lattice. Games and Economic Behavior. 7, 295-300. 